\title{
MicroRNA-124-3p affects myogenic differentiation of adipose- derived stem cells by targeting Caveolin-1 during pelvic floor dysfunction in Sprague Dawley rats
}

\author{
Hao Chen ${ }^{1}$, Zihao $\mathrm{Li}^{2}$, Ming Lin ${ }^{3}$, Xuling Lv ${ }^{1}$, Jingping Wang ${ }^{4}$, Qing Wei ${ }^{1}$, Zikai Zhang ${ }^{1}$, Liqun Li $^{4}$ \\ ${ }^{1}$ Department of Plastic Surgery, The First Affiliated Hospital of Wenzhou Medical University, Wenzhou, China; ${ }^{2}$ Hangzhou Medical College, \\ Hangzhou, China; ${ }^{3}$ Department of Obstetrics and Gynecology, The Second Affiliated Hospital of Wenzhou Medical University, Wenzhou, China; \\ ${ }^{4}$ Department of Plastic Surgery, The First Affiliated Hospital of Wenzhou Medical University, Wenzhou, China \\ Contributions: (I) Conception and design: H Chen; (II) Administrative support: Z Li; (III) Provision of study materials: Z Li, M Lin; (IV) Collection \\ and assembly of data: X Lv, J Wang; (V) Data analysis and interpretation: Q Wei, Z Zhang, L Li; (VI) Manuscript writing: All authors; (VII) Final \\ approval of manuscript: All authors. \\ Correspondence to: Liqun Li. Department of Plastic Surgery, The First Affiliated Hospital of Wenzhou Medical University, Wenzhou 325000 , China. \\ Email: liliqun9@aliyun.com.
}

Background: The aim of this study was to investigate using myogenic differentiation of adipose stem cells for the treatment of female pelvic floor dysfunction (PFD) and aimed to further study the influences of microRNA-124-3p (miR-124-3p) in the process of myogenic differentiation of adipose-derived stem cells (ADSCs) through targeting Caveolin-1 (Cav1) during PFD in Sprague Dawley (SD) rats.

Methods: The ADSCs were separated from 6-8-week-old female SD rats $(n=25)$ and were cultivated. Then, we observed the cell status and conducted fat and osteogenic experiments. We then constructed an ADSC-green fluorescent protein (GFP) stable transfer strain. Flow cytometry was used to identify the positive rates of CD44, CD90, and CD45 in ADSCs and ADSC-GFP. Real-time quantitative polymerase chain reaction (qRT-PCR) and western blotting were used to mRNA and protein expression levels. Myogenic differentiation of ADSCs was measured with immunofluorescence methods. A dual-luciferase reporter assay was executed to affirm whether Cav1 was a target of miR-124-3p.

Results: The isolated ADSCs cells were in good condition under the microscope. The results of flow cytometry showed that the positive rate of CD44 and CD90 was high, and the positive rate of CD45 was low in ADSCs and ADSC-GFP. Under normal culture conditions, ADSCs-GFP cells can be massively adipated and osteogenic. After 5-Aza induced ADSC-GFP myogenic differentiation, the level of miR-124$3 p$ was significantly increased. We found that MiR-124-3p mimics promoted the myogenic differentiation of ADSCs. Moreover, we discovered that Cav1 was a target gene of miR-124-3p and was negatively regulated by miR-124-3p. The results of leak point pressure (LPP), hematoxylin and eosin (HE), and Masson showed that the collagen fiber content of the PFD group was lower than that of the control group; the collagen fiber content of ADSC-GFP, 5-Aza, or miR-124-3p mimics were increased after intervention. Furthermore, the outcomes qRT-PCR, western blotting, and immunofluorescence suggested that miR-124-3p facilitated the survival ADSC-GFP fat transplantation by regulating many key factors in vivo.

Conclusions: These results proofed that miR-124-3p could accelerate myogenic differentiation of ADSCs by down-regulating Cav1 to improve PFD in SD rats, which will pave the way for therapeutic delivery of miRNA targeting PFD disease.

Keywords: Pelvic floor dysfunction; microRNA-124-3p; Caveolin-1; Sprague Dawley rats; adipose-derived stem cells (ADCs)

Submitted Nov 24, 2020. Accepted for publication Jan 19, 2021.

doi: $10.21037 /$ atm-20-8212

View this article at: http://dx.doi.org/10.21037/atm-20-8212 


\section{Introduction}

The female pelvic floor is a momentous organ in women. It has multiple functions in all humans, containing excretion of urine and feces, sex, and maintenance of pelvic organs (1). It is a complete anatomical structure, which is composed of nerves, muscles, and connective tissues (2). Studies have shown that vaginal delivery may cause various degrees of pelvic floor dysfunction (PFD) (3). Patients with PFD usually show a diverse range of clinical symptoms, including female genital prolapse and pain, lower urinary tract excretion and bowel dysfunction, and overactive bladder $(4,5)$. According to different definitions of the disease, more than $30 \%$ of women suffer from PFD, and the majority are adult women (6). The development of PFD is usually multifactorial, including obesity, race, age, pregnancy, childbirth, multiple births, chronic cough, history of pelvic surgery, genetics, spinal cord disease, and family history, among others $(7,8)$. There are both conservative and surgical treatments for PFD, and manifestations include overactive bladder syndrome, stress urinary incontinence and female reproductive organ prolapse (7). In the over 80 -year age group, $11 \%$ of PFD patients undergo surgery, which usually requires concurrent partial (anterior or posterior) or full vaginal repair and hysterectomy (9). Nevertheless, up to $20-30 \%$ of patients require repetitive surgery due to recurrence (10). Recently, the use of artificial biological mesh during surgical procedures has been shown to improve long-term recovery, but mesh can also cause pain, erosion, and scar formation in about $30 \%$ of cases (11). There is an urgent need to find new therapies to enhance the repair and regeneration of damaged tissues in PFD.

Female PFD usually manifests as abnormalities, injuries or defects in the female pelvic structure and symptoms include urinary incontinence, pelvic organ prolapse, etc. Drost et al. (12) indicated that the myogenic differentiation of human bone marrow-derived mesenchymal stem cells (MSCs) could be regarded as a latent treatment for urethral sphincter muscle repair. In a rat model of stress urinary incontinence, low-intensity pulsed ultrasound affected the myogenic differentiation of MSCs (13). Recently, studies have reported that stem cells have great potential in the treatment of PFD (14). Stem cells can promote tissue repair through cells that potentially differentiate into connective tissue relying on their multi-lineage differentiation ability (9). In recent years, adipose-derived stem cells (ADSCs) have attracted attention for repairing damaged tissues in clinical applications (15). In the human body, ADSCs are mesenchymal stem cells
(MSCs) originating from adipose tissue (16). In vitro, ADSCs have the ability to proliferate; widely distinguish adipogenesis, osteogenesis, cartilage and myogenic lineages, and secrete multifarious growth factors to stimulate tissue regeneration (17). In view of the above reports, it is necessary to conduct in-depth research on how ADSCs repair PFD.

MicroRNAs (miRNAs), consist of about 18-24 nucleotides, and are a series of small non-coding RNA that are concerned with modulation of post-transcriptional gene expression (18). It is recognized that miRNAs regulate the expression of about $30 \%$ of human proteins and participate in various biological processes, including cell immune and inflammatory responses, differentiation, proliferation, and apoptosis and migration, among others $(19,20)$. An increasing number of studies have indicated that miRNAs are important regulators of stem cell differentiation, regeneration, and development $(21,22)$. Expression profile analysis during myogenic differentiation or the development of stem cells has demonstrated various miRNAs with differential expression patterns, and these miRNAs may act as new myogenic modulators (23). Recently, a study revealed that $\mathrm{miR}-124-3 \mathrm{p}$ has important roles in the proliferation, differentiation, and regeneration of MSCs (24). However, the impacts of miR-124-3p on several biological processes, including muscle biology and myogenic differentiation of adipose stem cells, remain to be elucidated.

In the present study, we discovered that miR-124$3 p$ modulated the differentiation of ADSCs by targeting Caveolin-1 (Cav1), suggesting its potential roles in improving PFD.

We present the following article in accordance with the ARRIVE reporting checklist (available at http://dx.doi. org/10.21037/atm-20-8212).

\section{Methods}

\section{Isolation and culture of adipose stem cells}

Female Sprague Dawley (SD) rats (6-8 weeks old) from Cavens (Changzhou, China) were used for our experiment. The experimental protocol of our study was performed in accordance with the Guide for the Care and Use of Laboratory Animals and approved by the First Affiliated Hospital of Wenzhou Medical University. First, rats were anesthetized by injection of $15 \%$ chloral hydrate. Then, the rats were fixed on plates and disinfected with alcohol spray. The subcutaneous fat tissue of the rats' groin were cut and the visible blood vessels and fascia tissue were 
then removed. The tissues were washed with phosphate buffered saline (PBS), cut into small pieces, and placed in a centrifuge tube containing collagenase for digestion for $30 \mathrm{~min}$. After digestion, it was centrifuged and inoculated into a petri dish containing Dulbecco's Modified Eagle Medium (DMEM), antibiotics were added, and cultured at $37^{\circ} \mathrm{C}$ in a $5 \% \mathrm{CO}_{2}$ incubator.

\section{Cell transfection}

The miR-124-3p mimics and its corresponding negative control (NC) were obtained from RiboBio (Guangzhou, China) and were transfected into adipose stem cells (ADSCs) cells utilizing Lipofectamine 2000 reagents (Invitrogen, Carlsbad, CA, USA), following the supplier's instructions. The concentration of transfection was $50 \mathrm{~nm}$, and the solution was altered after 6 hours transient transfer. After 48 hours, the cells were subjected to qPCR for verification.

\section{qRT-PCR}

\section{qRT-PCR for miRNA}

Enriched miRNA was extracted via a miRNA isolation kit, following the manufacturer protocol (Omega, Norcross, GA, USA). The SYBR green stem-loop RT-PCR method (Takara Bio., Shiga, Japan) was used to quantify miRNAs expression levels. The housekeeping gene U6 served as the internal control for miRNA. Relative gene expression was measured by using the $2^{-\Delta \Delta \mathrm{Ct}}$ method.

\section{qRT-PCR for mRNA}

The total RNA of MH7A cells was isolated utilizing TRIzol reagent (Invitrogen, Carlsbad, CA, USA). A PrimeScript RT Reagent Kit (Takara Bio, Shiga, Japan) was utilized to synthesize complementary (c)DNA. Then, the mRNA expression of EIF2AK1 was analyzed through qPCR. This was conducted via a SYBR Premix Ex Taq II (Takara) kit on an ABI 7300 fast real time PCR system (Applied Biosystems, Foster City, CA, USA). The expression of glyceraldehyde 3-phosphate dehydrogenase (GAPDH) was regarded as an internal reference. The primers utilized for reverse transcription are shown in Table 1 .

\section{Western blotting analysis}

A radio-immunoprecipitation assay (RIPA) lysis buffer (Yisheng Biological Technology Co., Ltd., Shanghai, China) including proteinase suppressors was applied to isolate total protein. The concentration of total protein was detected by employing bicinchoninic acid (BCA) method (ComWin Biotech Co., Ltd., Beijing, China). A 10\% sodium dodecyl sulfate polyacrylamide gel electrophoresis (SDS-PAGE) was used to separate $20 \mu \mathrm{g}$ of protein samples. Next, the samples were put onto a polyvinylidene difluoride (PVDF) membrane. After being blocked with $5 \%$ non-fat milk for 1 hour, the membrane was incubated with the antibodies overnight at $4{ }^{\circ} \mathrm{C}$. The results were scanned by Quantity One software (version 4.6.9, Bio-Rad Laboratories, Hercules, CA, USA). The antibodies used in this part were as following, CD90 (Invitrogen Catalog \#MA180650); CD44 (Invitrogen Catalog \#MA5-17520); CD45 (Invitrogen Catalog \#12-0461-82); Cav1 (Bioss, bs-1453R); MyHC (proteintech, 22281-1-AP); Goat anti-rabbit cy3 (proteintech SA00009-2); GAPDH (proteintech, 60004-1Ig); MyoD (proteintech, 18943-1-AP); Collagen III (Bioss, bs-0549R); Collagen I (abcam, ab260043); LAMC1 (cell signaling, \#92921); MMP1 (Affinify, DF6325); MMP9 (abcam, ab76003); HOXA11 (Bioss, bs-666R); Elastin (Bioss, bs-1756R); Calponin (abcam, ab46794); Vimentin (abcam, ab92547); GFP (abcam, ab1218).

\section{Establishment of green fluorescent protein stable transfection ADSC cell line}

The ADSCs cells were plated in a $3.5 \mathrm{~cm}$ dish with a plating density of $50 \%$. After 24 hours of cell attachment, the multiplicity of infection (MOI) value was 10 for green fluorescent protein (GFP) virus (Hanbio, Shanghai, China) infection. After $72 \mathrm{~h}$ of infection, fresh medium was replaced and puromycin (cat no. X10020; XYbio; Hangzhou; China) was added at a concentration of $5 \mu \mathrm{g} / \mathrm{mL}$. Then, the cell fluorescence and status were observed under a fluorescence microscope. After puromycin screening, there were some cells death in the beginning and the fluorescence was weaker. With prolongation of the selection time, the status of cells was improved; the fluorescence was increased and could be passaged stably, indicating that a stable transgenic strain of ADSCs-GFP had been successfully constructed.

\section{Flow cytometry analysis of ADSCs surface markers}

The ADSCs were harvested by trypsinization and resuspended in PBS containing 4\% fetal bovine serum (FBS). Cells were then stained with fluorescein isothiocyanate (FITC)-conjugated anti-human CD44, CD90, and CD45 antibodies (eBioscience, Inc., San Diego, 
Table 1 The primers used in qRT-PCR

\begin{tabular}{|c|c|}
\hline Gene (R) & Sequence \\
\hline \multirow[t]{2}{*}{$\operatorname{miR}-124-3 p$} & F: ACACTCCAGCTGGGTAAGGCACGCGGTG \\
\hline & $\begin{array}{l}\text { R: CTCAACTGGTGTCGTGGAGTCGGCAATTCA } \\
\text { GTTGAGGGCATTCA }\end{array}$ \\
\hline \multirow[t]{3}{*}{$\operatorname{miR}-140-5 p$} & F: ACACTCCAGCTGGGCAGTGGTTTTACCCTA \\
\hline & R: CTCAACTGGTGTCGTGGAGTCGGCAATTCA \\
\hline & GTTGAGCTACCATA \\
\hline \multirow[t]{2}{*}{$m i R-194-5 p$} & F: ACACTCCAGCTGGGTGTAACAGCAACTCCA \\
\hline & $\begin{array}{l}\text { R: CTCAACTGGTGTCGTGGAGTCGGCAATTCA } \\
\text { GTTGAGTCCACATG }\end{array}$ \\
\hline \multirow[t]{2}{*}{$\operatorname{miR}-129-5 p$} & F: ACACTCCAGCTGGGCTTITTGCGGTCTGG \\
\hline & $\begin{array}{l}\text { R: CTCAACTGGTGTCGTGGAGTCGGCAATTCA } \\
\text { GTTGAGGCAAGCCC }\end{array}$ \\
\hline \multirow[t]{2}{*}{$\operatorname{miR}-132-3 p$} & F: ACACTCCAGCTGGGTAACAGTCTACAGCCA \\
\hline & $\begin{array}{l}\text { R: CTCAACTGGTGTCGTGGAGTCGGCAATTCA } \\
\text { GTTGAGCGACCATG }\end{array}$ \\
\hline \multirow[t]{2}{*}{$m i R-181-5 p$} & F: ACACTCCAGCTGGGAACATTCAACGCTGTCG \\
\hline & $\begin{array}{l}\text { R: CTCAACTGGTGTCGTGGAGTCGGCAATTCA } \\
\text { GTTGAGACTCACCG }\end{array}$ \\
\hline \multirow[t]{2}{*}{ miR-199-5p } & F: ACACTCCAGCTGGGCCCAGTGTTCAGACTAC \\
\hline & $\begin{array}{l}\text { R: CTCAACTGGTGTCGTGGAGTCGGCAATTCA } \\
\text { GTTGAGGAACAGGT }\end{array}$ \\
\hline \multirow[t]{2}{*}{ U6 } & F: CTCGCTTCGGCAGCACA \\
\hline & R: AACGCTTCACGAATTTGCGT \\
\hline \multirow[t]{2}{*}{ Myog } & F: CATCCСССТАТTTСТАСCA \\
\hline & R: TTACACACCTTACACGCCC \\
\hline \multirow[t]{2}{*}{ MyHC } & F: GGTGGTGGAAAGAAAGGG \\
\hline & $\begin{array}{l}\text { R: CAAAGTGGGGGTGAGTGCCTCAACTGGTGT } \\
\text { CGTGGAGTCGGCAATTCAGTTGAGACTCACCG }\end{array}$ \\
\hline \multirow[t]{2}{*}{ GAPDH } & F: ACAGCAACAGGGTGGTGGAC \\
\hline & R: TTTGAGGGTGCAGCGAACTT \\
\hline
\end{tabular}

qRT-PCR, real-time quantitative polymerase chain reaction; GAPDH, glyceraldehyde 3-phosphate dehydrogenase.

CA, USA). Flow cytometry was carried out by FACSDiva (Canto, Becton, Dickinson and Co. Biosciences, San Jose, CA, USA), and FACS analysis was executed utilizing FlowJo software (Tree Star, Ashland, OR, USA).

\section{Dual-luciferase reporter assay}

After $48 \mathrm{~h}$ of transfection, the cells were seeded into 24- well plates. After incubation for $24 \mathrm{~h}$, the construction of a dual fluorescein reporter vector was achieved by the 3'UTR of wild type (WT) and mutant (MUT) Cav1 being cloned into pGL3-RB-REPORTTM. The miR-124-3p mimic and PGL3-3'UTR-WT or PGL3-3'UTR-MUT were cotransfected into cells by applying Lipofectamine 2000 reagent (Invitrogen, Carlsbad, CA, USA). Finally, the luciferase activity was assessed using the Dual-Luciferase Reporter Assay System (Promega Corp., Madison, WI, USA).

\section{Lipidation experiment}

After the ADSC-GFP cells were expanded and cultured, they were collected and seeded in a 24-well plate pre-coated with gelatin. Each well was added with $1 \mathrm{~mL}$ DMEM highsugar complete medium and cultured at $37{ }^{\circ} \mathrm{C}, 5 \% \mathrm{CO}_{2}$. When the cells had adhered to the wall and grown about $90 \%$, the medium was changed to lipid induction medium for culture. The induction solution was changed every 3 days. After 14 days, Oil red O staining was carried out according to the instructions.

\section{Oil red $O$ staining}

The ADSCs were cultivated in DMEM and gathered on the 7 th day and 14th day, respectively. The cells were fixed with $10 \%$ formalin, washed with $60 \%$ isopropanol, and stained with Oil red $\mathrm{O}$ working fluid. Then, after being fixed in glycerin gelatin, the cells were observed under a microscope (Olympus optics, Tokyo, Japan). Finally, the results were counted under the microscope.

\section{Osteogenesis experiment}

After the ADSC-GFP cells were expanded and cultured, the cells are collected and seeded in a 24-well plate pre-coated with gelatin. Each well received $1 \mathrm{~mL}$ of DMEM highsugar complete medium and was cultured at $37^{\circ} \mathrm{C}, 5 \% \mathrm{CO}_{2}$. When the cells had adhered to the wall and grown about $90 \%$, they were transferred to an osteoinduction medium for culture. The induction solution was changed every 3 days. After 2 weeks, alizarin red staining was performed following the manufacturer's instructions.

\section{Alizarin red staining}

The ADSC cells $\left(2 \times 10^{4} / \mathrm{mL}\right)$ were added into 24 -well plates and cultured under normal conditions. Every 3 days, the 
medium was replaced. After 2 weeks of incubation, the cells were washed twice with PBS and stained with $0.2 \%$ alizarin red for 20 minutes. Finally, the cells were washed with distilled water, air-dried at room temperature, resuspended in $10 \%$ acetic acid, and quantified spectrophotometrically at a wavelength of $450 \mathrm{~nm}$.

\section{Rat PFD model}

A total of 25 female SD rats, 6-8 weeks old, were purchased from Changzhou Cavens Company (Changzhou, China) and divided into 5 groups (each group 5 rats). An $18 \mathrm{f}$ catheter was inserted into the rat's vagina, and fixed with a single 3-0 silk thread. A Foley balloon was filled with water $(2.5-3.0 \mathrm{~mL})$ and connected to a pressure sensor to generate pressure on the pelvic support tissue. After 4 hours, the catheter was fetched and removed from the vagina together with the pressure sensor. At 14 days after vaginal dilation, the leak point pressure (LPP) was tested to ensure establishment of the PFD model.

\section{LPP test}

The cells were injected into groups according to the experiment and kept for 7 days. Rats in each group were tested for LPP. Two days before the LPP test, a bladder catheter was interposed into the PFD rat and attached to the pressure transducer and micro-infusion pump through a shut-off valve. Under anesthesia with urethane, the bladder was palpated to empty and filled with normal saline at a rate of $5 \mathrm{~mL} /$ hour through the micro-infusion pump. Pressure was increased gradually until the rat leaked saline through the urethra. When the first signs of urethral leakage appeared, the external abdominal pressure was quickly removed. In the absence of detrusor muscle contraction, the peak pressure LPP at the time of leakage was recorded. The LPP was counted by subtracting the bladder baseline pressure from peak bladder pressure. The bladder was drained and refilled, and the process was repeated 3 times in each rat. Finally, mean bladder baseline pressure and mean LPP were counted for each rat.

\section{Hematoxylin and eosin (HE) and Masson staining}

After the in vitro tests were completed, complete urethra, vagina and fascia tissue, and bladder tissue were extracted and fixed with $4 \%$ paraformaldehyde (PFA) overnight. The urethra and surrounding tissues were dehydrated, inserted in paraffin wax, incised into $5 \mu \mathrm{m}$ sections and dewaxed usually. Then, HE and Masson staining were carried out. For the HE staining, the tissues were stained with hematoxylin for 5 minutes, washed with distilled water until they showed blue-purple, and then stained with eosin. Next, the tissue sections were rinsed with distilled water and dehydrated with absolute ethanol. Finally, neutral gum was added to the tissue sections and they were sealed. For the Masson staining, the tissues were firstly washed with PBS and then stained with the prepared weight iron hematoxylin staining solution for 5-10 min. Then, the acidic ethanol differentiation solution was used to differentiate for 5-15 s; Masson blue solution was utilized to return blue for 3-5 min and washed with distilled water for $1 \mathrm{~min}$. Next, the tissues were stained with Ponceau red magenta staining solution for 5-10 min. Finally, neutral gum was added to the tissue sections and they were sealed. Tissue histomorphology was surveyed and photographed under a light microscope.

\section{Immunofluorescence staining}

\section{Immunofluorescence detection of cells}

After 2 weeks of fibroblast differentiation of ADSCs, myosin heavy chain $(\mathrm{MyHC})$ expression was tested by immunofluorescence. The cells were fixed in 4\% PFA for 15-20 minutes, washed with PBS 3 times, made to be transparent in PBS with $0.2 \%$ Triton $\mathrm{X}-100$ for 20 minutes, and closed with bovine serum albumin (BSA). After being washed with PBS, the cells were hatched with rabbit anti-rat collagen I overnight at $4{ }^{\circ} \mathrm{C}$, and they were further incubated with Alexa488-labeled goat anti-rabbit IgG antibody at room temperature in the dark for 1 hour, mounted with $50 \%$ glycerin, and photographed under a fluorescence microscope.

\section{Tissue immunofluorescence detection}

The tissues slices were soaked in the antigen retrieval solution and microwaved for 20 minutes on high heat. Then, PBS was used to wash the slice surface and repeated 3 times for 5 minutes. After the tissue slices were incubated with $\mathrm{H}_{2} \mathrm{O}_{2}$ for 10 minutes at room temperature, they were blocked with $5 \%$ BSA at $37{ }^{\circ} \mathrm{C}$ for 1 hour. After dilution, they were incubated with the primary antibodies at $37{ }^{\circ} \mathrm{C}$ for 2 hours, and then hatched with secondary antibodies at $37^{\circ} \mathrm{C}$ for 1 hour. Lastly, the tissue slices were stained with Hoechst at room temperature for 15 minutes. The results were observed and photographed under a microscope. 


\section{Data statistics}

All data statistics were executed by the statistical software Graphpad 8.0 (San Diego, CA, USA) and SPSS 22.0 (IBM, Chicago, IL, USA). The differences between 2 groups were determined by the Student's t-test. The differences among 3 or more than 3 groups were analyzed via one-way analysis of variance (ANOVA) with the Bonferroni post hoc test. Statistical difference was considered at $\mathrm{P}<0.05$.

\section{Results}

\section{ADSCs were separated successfully from rats}

After 24 hours, the primary culture medium was replaced, and the adherent cells revealed a polygonal or elongated shape, with anomalous morphology, a large nucleus, less cytoplasm, and intense light refraction (Figure 1A). Along with the increase of culture time, these cells became larger and longer with more of a spindle-shape, and the cell colonies were formed. The cells continued to proliferate in the colonies. After about 5-7 days, the bottom of the culture dish was covered with cells, and the cells were arranged in a spiral shape (Figure $1 B$ ). We used passage to purify cells, which made them long and fuse. After more than 3 repetitions of purification and culture, there was almost no change in cell shape, indicating that it had been passaged stably (Figure 1C). The expression of CD44, CD90, and CD45 in ADSCs was tested through flow cytometry. The outcomes showed that CD44 and CD90 were highly expressed, while CD45 was lowly expressed, in ADSCs (Figure 1D). These findings demonstrated that the ADSCs had been successfully isolated from rats.

\section{Establishment of GFP stable transfection ADSC cell line as well as adipogenic and osteogenic experiments}

Flow cytometry was used to verify the successful construction of ADSCs-GFP. As illustrated in Figure 1E, flow cytometry showed that the positive rates of CD44, CD90, and CD45 were $97.8 \%, 99.2 \%$, and $0.422 \%$, respectively, indicating that $\mathrm{CD} 44$ and $\mathrm{CD} 90$ were highly expressed, whilst CD45 was silenced in the ADSCs-GFP. We then used Oil Red O staining to analyze the fat-forming experiment of ADSCs-GFP. It was revealed (Figure $1 F$ ) that ADSCs-GFP cells could be massively adipated under normal culture conditions. Besides, we also used Alizarin Red staining to analyze the osteogenesis experiment of ADSCs-GFP. The results of Figure $1 G$ show that ADSCs-
GFP cells could be prone to osteogenic differentiation under normal conditions. Moreover, we used 5 -azacytidine (5-aza) to induce the myogenic differentiation of ADSCGFP to further verify the successful verification of ADSCGFP. The myogenic differentiation was shown in Figure $1 \mathrm{H}$. The fluorescence images and white light images taken by the microscope after stable transfection of GFP into ADSC cells were displayed in Figure $2 A, B$. Besides, the flow cytometry of GFP expression was showed in Figure 2C. Furthermore, the qRT-PCR results showed that the expression of miR124-3p, miR-140-5p, and miR-181-5p were significantly increased after 5-Aza induced ADSC-GFP myogenic differentiation, compared with the ADSCs-GFP group (Figure 2D-7). Above all, this section of results revealed that the ADSCs-GFP model was successfully established and it was prone to osteogenic and lipid differentiation.

\section{MiR-124-3p mimics promote the myogenic differentiation of ADSCs}

To explore the influences of miR-124-3p on ADSCs, we transfected miR-124-3p mimics into ADSCs and enticed myogenic differentiation. After 48 hours transfection, the levels of myogenic markers, containing Cav1, Myog, and MyHC, were analyzed by qRT-PCR and immunofluorescence to verify the transfection efficiency. As shown in Figure 3A, miR-124-3p was up-regulated in ADSCs. Besides, miR-124-3p facilitated myogenesis in ADSCs, leading to the increased expression of Myog and $\mathrm{MyHC}$, the decreased expression of Cav1, as revealed by by qRTPCR (Figure 3B-D). In addition, immunofluorescence results showed that, compared with the NC group, the expression of $\mathrm{MyHC}$ in the miR-124-3p mimics group was significantly fortified, and the expression of Cav1 was significantly reduced (Figure 3E,F). Together, these consequences suggested that miR-124-3p promoted myogenesis in ADSCs.

\section{MiR-124-3p promotes the myogenesis of ADSCs by targeting and regulating the expression of Cav 1}

We used the target prediction algorithm TargetScan (www. targetscan.org) to probe the potential target genes of miR124-3p. Finally, we discovered that Cav1 was one of the target genes of miR-124-3p (Figure 4A). Besides, Figure $4 B$ elucidates that the introduction of miR-124-3p mimic weakened the luciferase activity of cells containing Cav1 3'UTR-WT instead of Cav1 3'UTR-MUT, compared with mimic NC group. The qPCR, western blotting, and 
A

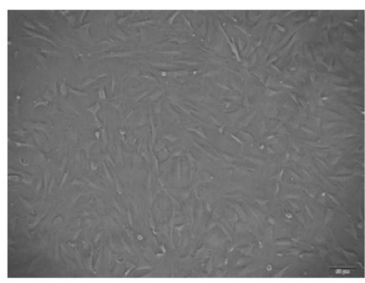

D

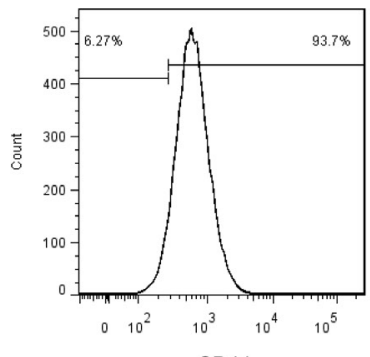

$\mathrm{E}$

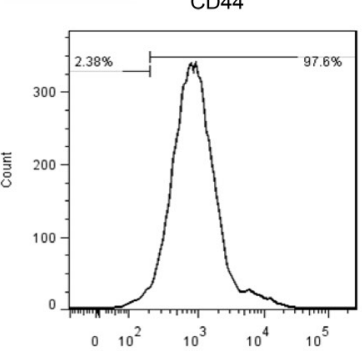

$\mathrm{F}$

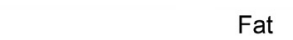

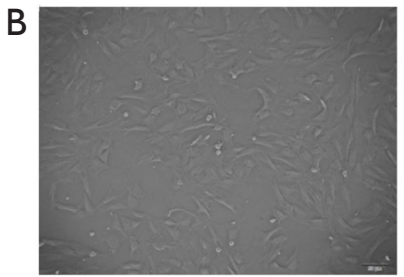

CD90
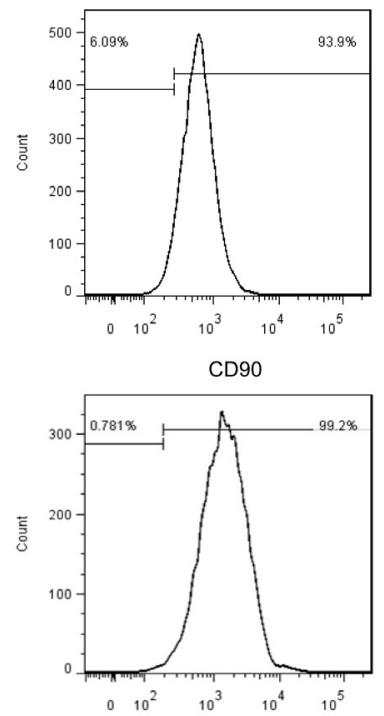

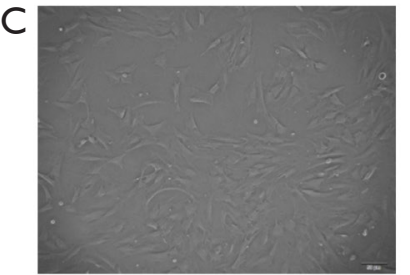

CD45

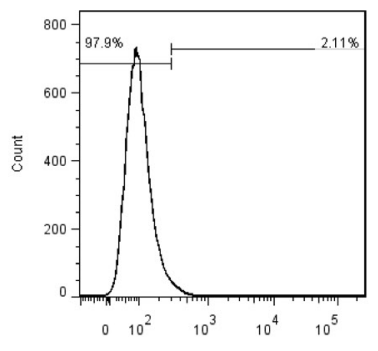

CD45

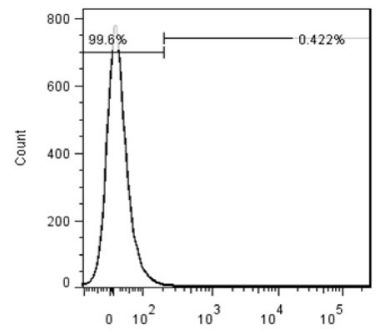

G

Osteogenesis

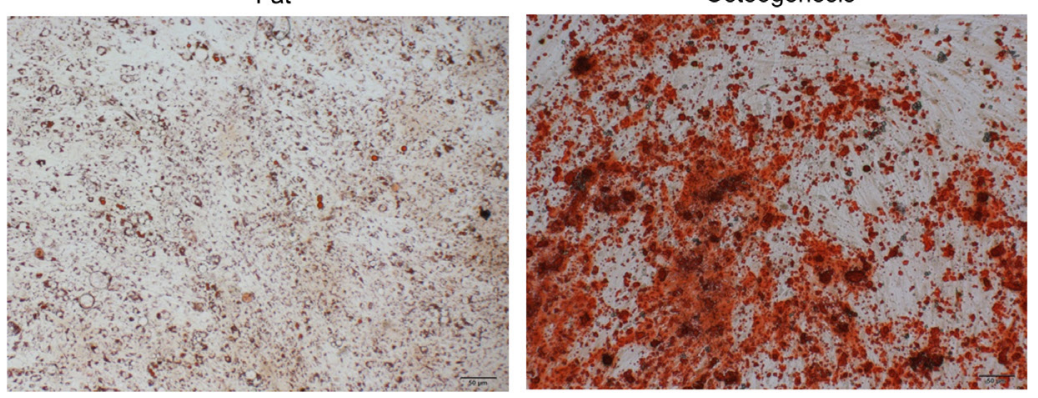

$\mathrm{H}$ desmin hoechst
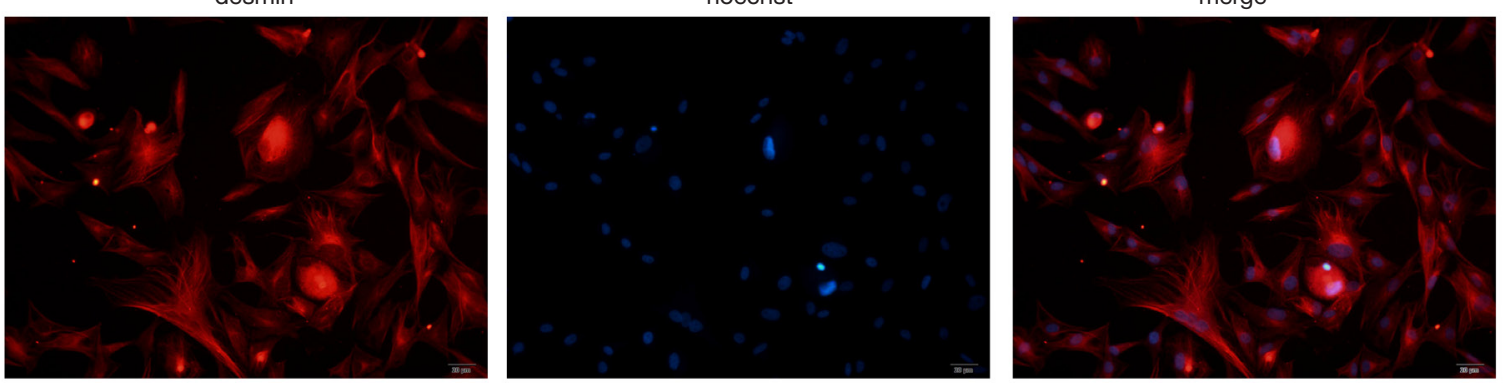

Figure 1 Isolation and identification of ADSCs and the successful construction of the ADSC-GFP cell line. (A) The condition of primary ADSCs after adherent growth for 24 hours; (B) the condition of ADSC primary cultures at the 6th day; (C) the condition of the third-generation ADSCs after being cultured for 7 days; (D) the expression of surface markers CD44, CD90, and CD45 in the thirdgeneration ADSCs was tested by flow cytometry; (E) the expression of surface markers CD44, CD90, and CD45 in the third-generation ADSCs was detected by flow cytometry; (F) after adipogenic induction, the Oil red O staining for the third-generation ADSCs; (G) after osteogenic differentiation, the Alizarin red staining for the third-generation ADSCs. $(\mathrm{H})$ The myogenic differentiation was tested by immunofluorescence. Amplification of (A-C), (F-G): 100x; scale: 20 um. ADSCs, adipose-derived stem cells. 
A

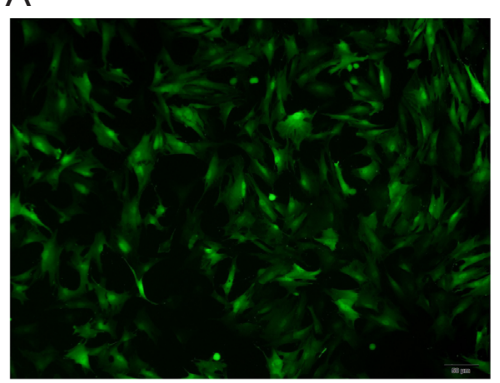

B

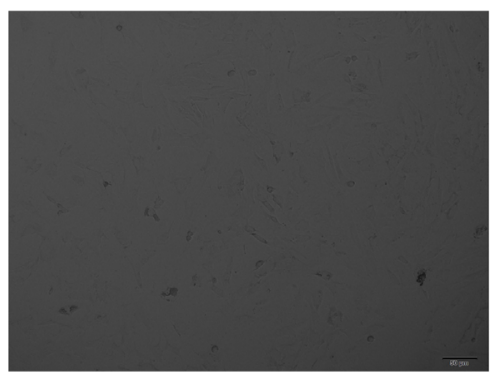

C

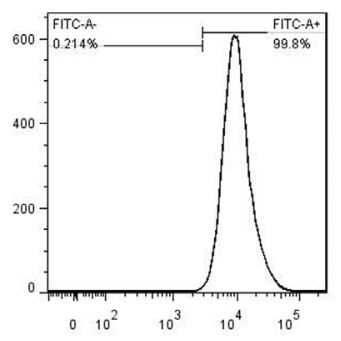

G
$\mathrm{D}$

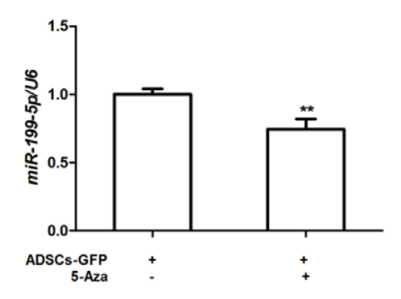

$\mathrm{H}$
$E$

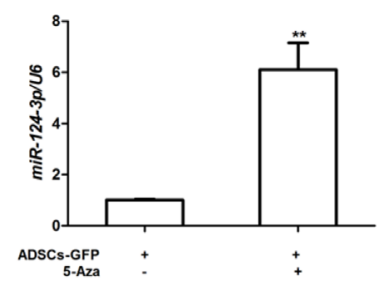

I
$\mathrm{F}$

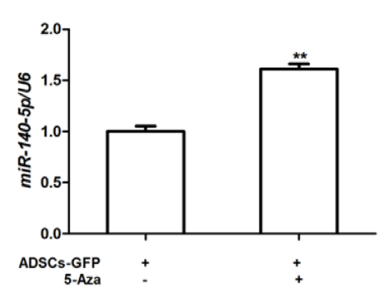

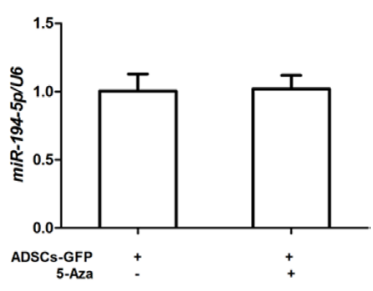

J

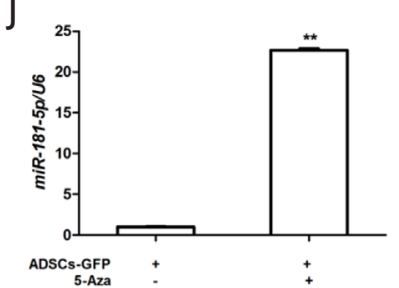

Figure 2 The mRNA expression of key miRNAs after 5-Aza induces ADSC-GFP myogenic differentiation. The fluorescence images (A) and white light images (B) taken by the microscope after stable transfection of GFP into ADSC cells were displayed. (C) The expression of GFP was tested by flow cytometry. Through qRT-PCR, the mRNA expression levels of miR-199-5p (D), miR-124-3p (E), miR-140-5p (F), miR-194-5p (G), miR-129-5p (H), miR-132-3p (I), miR-181-5p (J) were analyzed. ${ }^{* *} \mathrm{P}<0.01$ vs. ADSCs-GFP group. ADSCs-GFP, adiposederived stem cells-green fluorescent protein; qRT-PCR, real-time quantitative polymerase chain reaction.

immunofluorescence analyses illustrated that the expression of MyoG, MyoD, and MyHC in the miR-124-3p mimic group was significantly increased, whilst the expression of Cav1 was significantly reduced, compared with the miR-124-3p mimic NC group (Figure 4C,D). Moreover, compared with the ADSC-GFP, $10 \mu \mathrm{mol} / \mathrm{L}$ 5-Aza, or empty vector group, the expression levels of $\mathrm{MyoG}, \mathrm{MyoD}$, and $\mathrm{MyHC}$ in the Cav1 overexpression group decreased while the expression levels of Cav1 increased (Figure 4C-E). The results of this section implied that miR-124-3p directly targeted Cav1 to affect its expression on ADSCs.

\section{Results of LPP test, HE, and Masson staining of urethral and surrounding tissues in each group}

After construction of the PFD rat model, the LPP in the
PFD model group were found to be smaller than those in the Control group, indicating that the PFD models had been successfully established (Figure 5A). After 4 weeks of ADSC-GFP transplantation, the LPP in the PFD group were significantly increased. Furthermore, the intervention of 5-Aza and miR-124-3p mimics could also increase the value of LPP (Figure $5 A$ ).

In the Control group, the results of HE staining suggested that the arrangement of muscle fibers in urethral and surrounding tissues was intensive, with a complete muscular layer and no rupture, and was pink in color. Nevertheless, the muscular layer of the urethral wall was destroyed, thin, loose, deranged, and shrunken in the PFD model group. Transplantation of ADSC-GFP could distinctly boost the arrangement and density of muscle fibers to tighten the muscular layer and connective tissues of the fascia in 
A

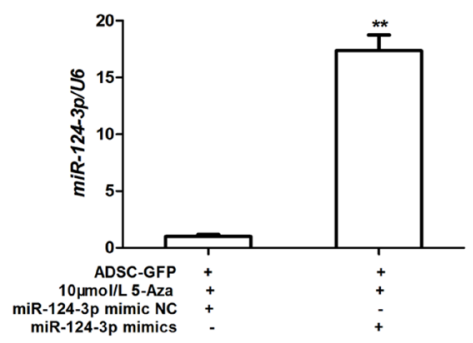

B

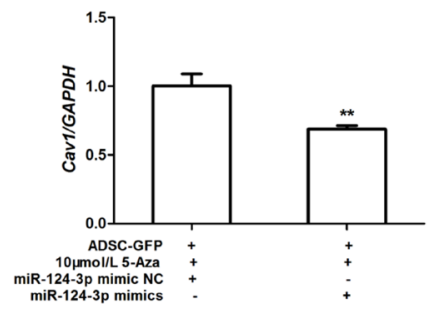

C

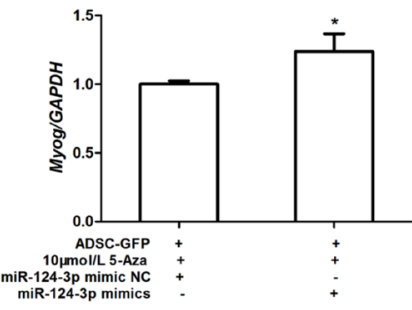

D

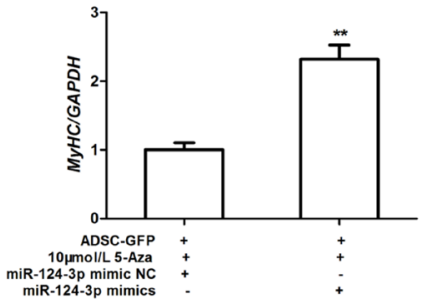

$\mathrm{E}$
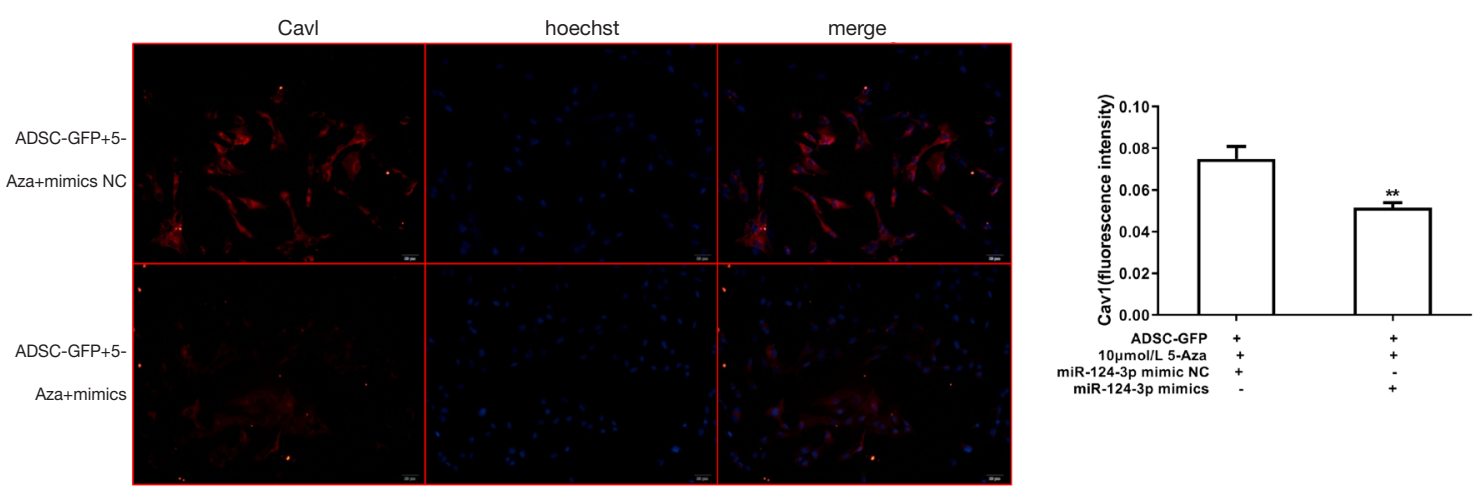

$\mathrm{F}$
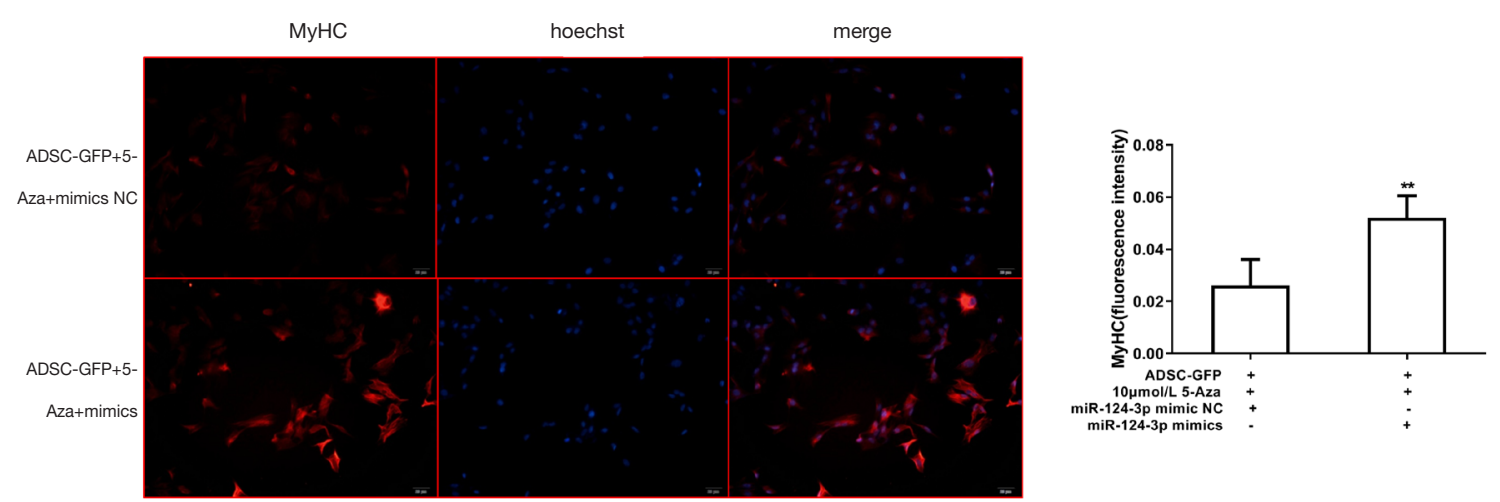

Figure 3 MiR-124-3p over-expression accelerates the myogenic differentiation of ADSCs. The expression of miR-124-3p (A), Cav1 (B), Myod (C), and MyHC (D) were tested by qRT-PCR. The expression of Cav1 (E) and MyHC (F) were detected by immunofluorescence. ADSCs, adipose-derived stem cells. ${ }^{*} \mathrm{P}<0.05,{ }^{* *} \mathrm{P}<0.01$ vs. ADSCs-GFP group.

the PFD group. Additionally, the muscle fiber structure was completely repaired in the group co-transfected with 5-Aza and miR-124-3p mimics. It was also revealed that 5-Aza could complete the reparative influence of ADSCs on the muscle fiber structure in the PFD group (Figure 5B).

Masson staining led to collagen fibers that were stained blue in color with homogeneous staining and a larger stained area. In the PFD group, the collagen fibers were stained light blue, loose and deranged, and the proportion of connective tissues was obviously fortified. The partial vascular wall was thickened with hyalinization. After ADSC-GFP transplantation, collagen staining was boosted with a regular arrangement. The co-transfection of miR-124-3p mimics and 5-Aza could boost collagen expression, facilitate the repair of structure and tighten the muscular layer and fascia. The 5-Aza could inhibit the impact of ADSCs on the repair of the collagen structure in the PFD group (Figure 5C).

\section{The fascia formation-associated protein expression in rat uretbra and surrounding tissues}

The RT-PCR results revealed that, compared with the 
A

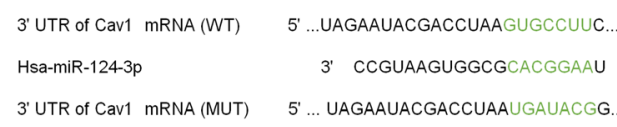

B

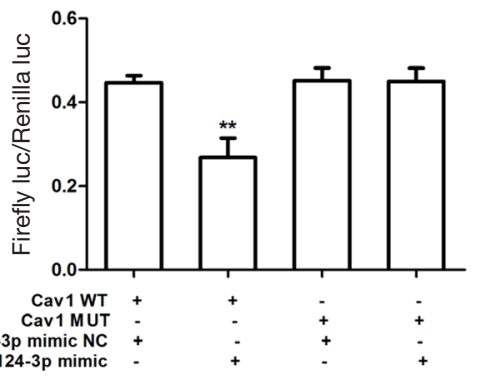

C

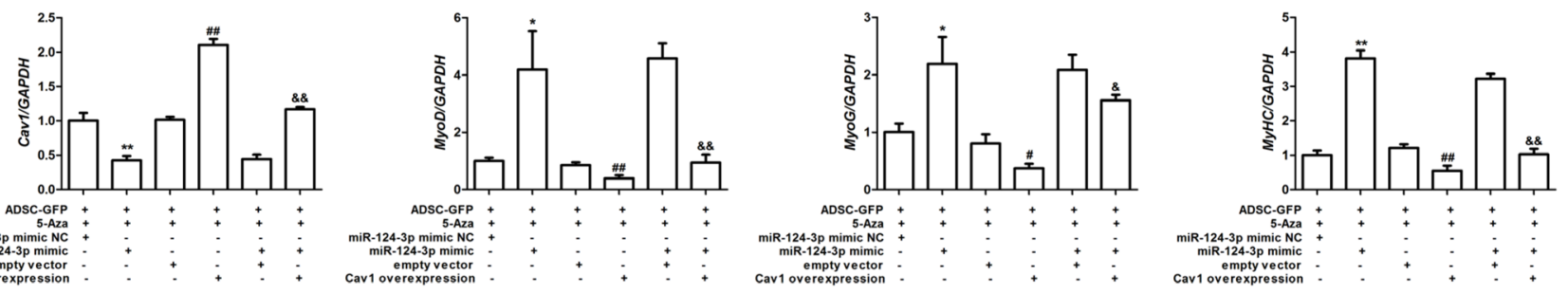

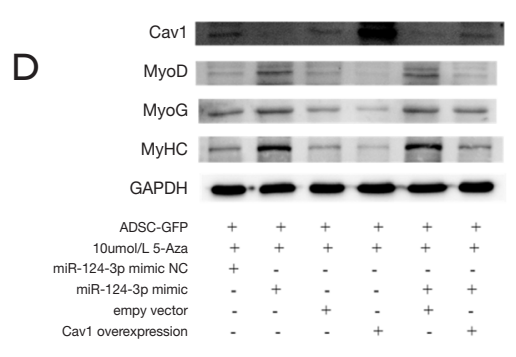

$E$

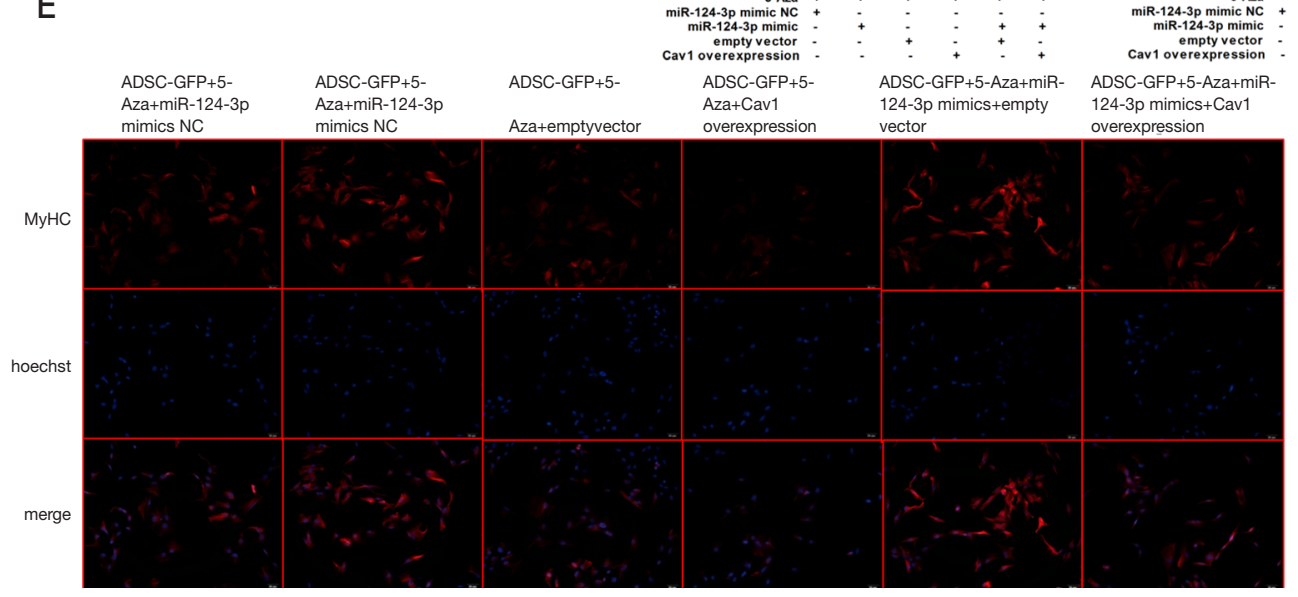

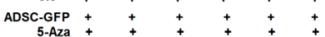

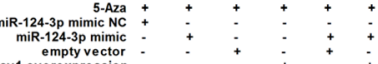
mempty vector
eav1 overexpression

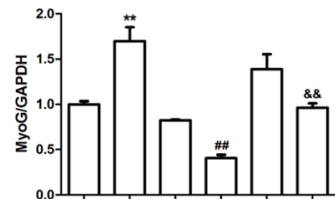

ASC-GFP

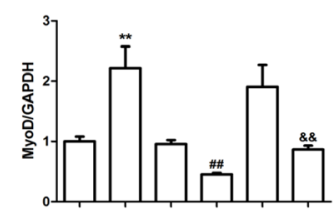

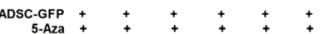
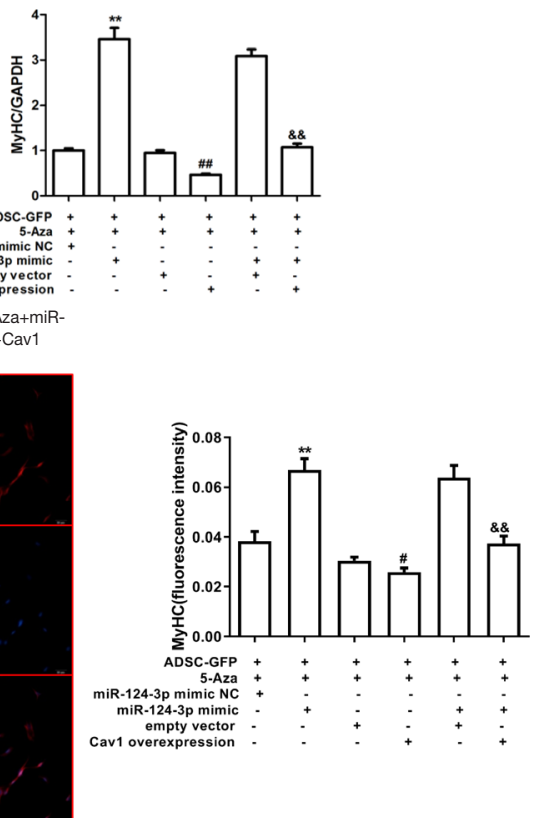

Figure 4 Cav1 is a target of miR-124-3p. (A) The potential binding sites between miR-124-3p and Cav1. (B) Luciferase reporter activity in ADSCs transfected with WT, MUT 3'-UTR of Cav1. ${ }^{* *} \mathrm{P}<0.01$ vs. mimic NC. (C) qRT-RCR reveals mRNA expression levels of Cav1, Myod, Myog, and MyHC in ADSCs. (D) Western blot reveals the protein levels of Cav1, Myod, Myog, and MyHC in ADSCs. (E) The expression of $\mathrm{MyHC}$ was detected by immunofluorescence. $(\mathrm{C}-\mathrm{E}){ }^{*} \mathrm{P}<0.05,{ }^{* *} \mathrm{P}<0.01$ vs. ADSC-GFP; ${ }^{*} \mathrm{P}<0.05,{ }^{* \#} \mathrm{P}<0.01$ vs miR-124-3p mimic NC; ${ }^{\text {\&\& }} \mathrm{P}<0.01$ vs. empty vector. qRT-PCR, real-time quantitative polymerase chain reaction; ADSCs, adipose-derived stem cells. 


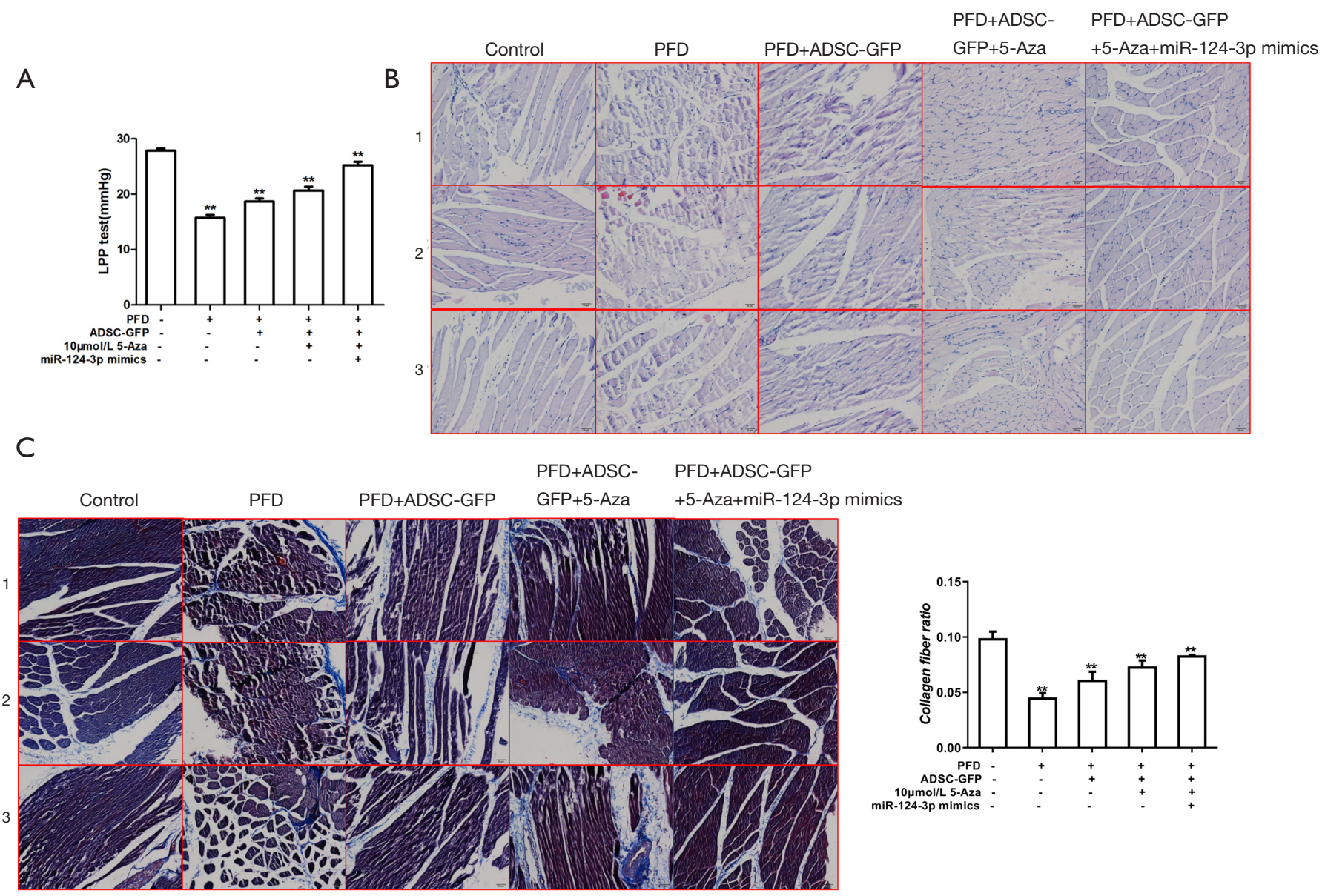

Figure 5 Animal modeling experiment. Results of LPP (A), HE (B), and Masson staining (C) in the urethra and surrounding tissues among the 5 groups. Amplification, 100x; scale, $20 \mu \mathrm{m}$. LPP, leak point pressure; HE, hematoxylin and eosin. ${ }^{* *} \mathrm{P}<0.01$ vs. PFD.

Control group, the expression of miR-124-3p, desmin, calponin, vimentin, elastin, Col1a1, Col3a1, and HOXA11 in the PFD model group was significantly reduced, and the expression of Cav1, LAMC1, MMP1, and MMP9 were significantly increased (Figure 6). The interventions of ADSC-GFP, 5-Aza, and miR-124-3p mimics could increase the expression of miR-124-3p, desmin, calponin, vimentin, elastin, Col1a1, Col3a1, and HOXA11, while reducing the levels of Cav1, LAMC1, MMP1, and MMP9 (Figure 6).

Western blotting results illustrated that, relative to the Control group, the expression of Elastin, Collagen I, Collagen III, and HOXA11 in the PFD model group were visibly reduced, whilst the expression of Cav1, LAMC1, MMP1, and MMP9 were significantly increased (Figure 7). We found that ADSC-GFP, 5-Aza, and miR-124-3p mimics could increase the expression of elastin, Collagen I, Collagen III, and HOXA11, but decrease the expression of
Cav1, LAMC1, MMP1, and MMP9 (Figure 7).

Immunofluorescence results showed that vimentin, desmin, and calponin expression levels in the PFD model group were significantly lower than those in the Control group (Figure 8A,B). The ADSC-GFP, 5-Aza, and miR124-3p mimics could all increase the expression of vimentin, desmin, and calponin (Figure 8A,B). However, the content of GFP were not significantly different among the groups (Figure 8C). Immunofluorescence results showed that Collagen III and Elastin expression levels in the PFD model group were significantly lower than those in the Control group (Figure 8D,E). The ADSC-GFP, 5-Aza, and miR124-3p mimics could all increase the expression of Collagen III and Elastin (Figure 8D,E). Together, these outcomes suggested that miR-124-3p facilitated the survival ADSCGFP fat transplantation by regulating many key factors in vivo. 
A

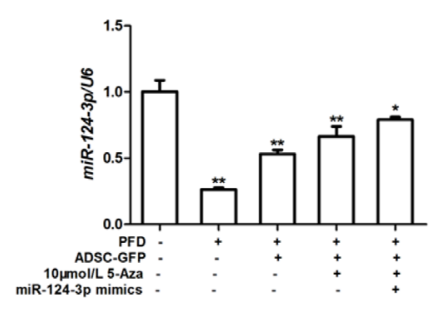

E

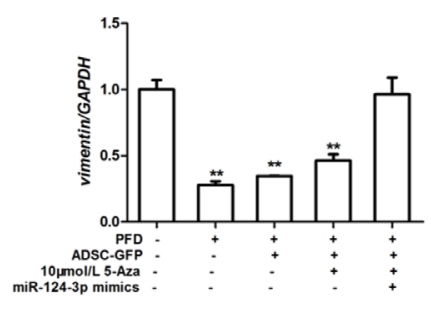

I

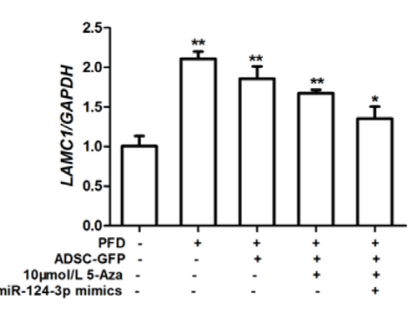

B

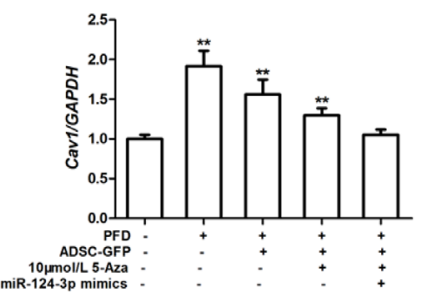

C

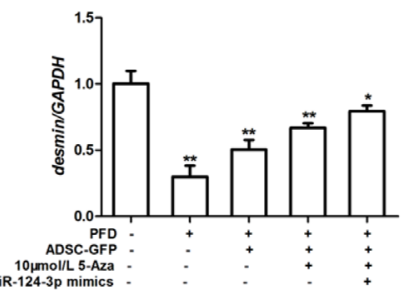

D

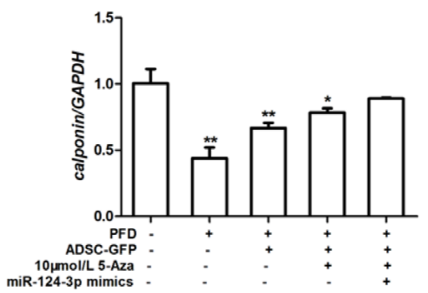

$\mathrm{F}$

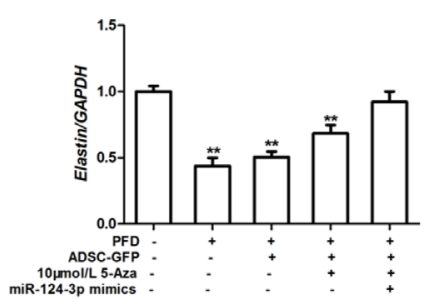

$J$
G

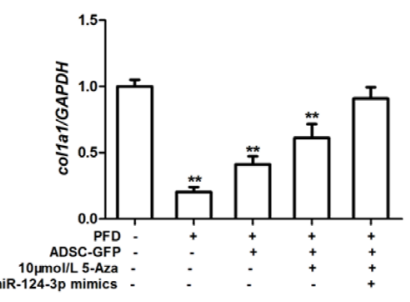

$\mathrm{K}$

$\mathrm{H}$

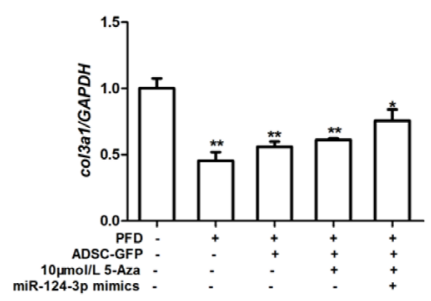

L

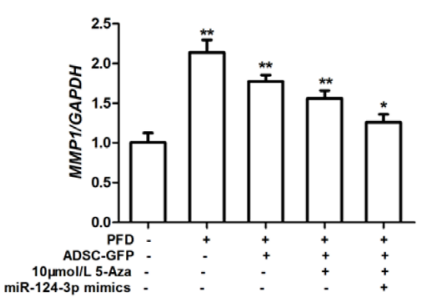

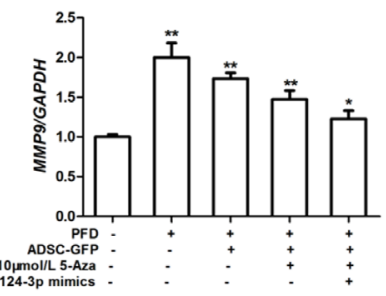

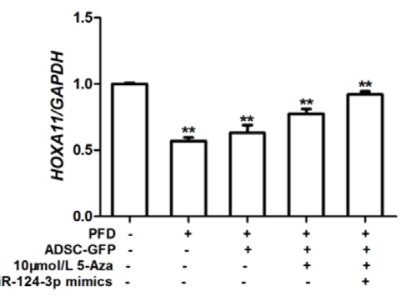

Figure 6 The mRNA expression of fascia formation-related genes in the urethra and surrounding tissues were analyzed by qRT-PCR. The mRNA expression of miR-124-3p (A), Cav1 (B), desmin (C), calponin (D), vimentin (E), elastin (F), Col1a1 (G), Col3a1 (H), LAMC1 (I), MMP-1 (J), MMP-9 (K), HOXA11 (L). qRT-PCR, real-time quantitative polymerase chain reaction. ${ }^{*} \mathrm{P}<0.05,{ }^{* *} \mathrm{P}<0.01$ vs. $\mathrm{PFD}$.

\section{Discussion}

The incidence of PFD is high among adult women (25). Stem cells can be easily isolated and expanded from various adult tissues, and the transplantation of ADSCs has been a goal of PFD treatment (26). Additionally, certain miRNAs have been shown to positively modulate $\operatorname{ADSCs}(27,28)$. Hence, the aim of our study was to probe the impacts of miR-124-3p-modified ADSCs on PFD recovery.

Many previous studies have demonstrated some functions of ADSCs, including that ADSCs could be successfully separated and ADSCs represented apoptosis potential for adipogenic and osteogenic differentiation $(29,30)$. In line with the above, our study also discovered these kinds of functions in ADSCs. Besides, bone marrow mesenchymal stem cell (BMSC) transplantation has been shown to enhance the LPP in a rat model of PFD (31). In a rat model, the function of bladder was able to be enhanced by MSC injection (32). In our study, we discovered that miR-124-3p, miR-140-5p, and miR-181-5p were highly expressed in ADSCs. Guo et al. (33) revealed that miR140-5p silencing facilitated osteogenesis of ADSCs through targeting BMP2 and TLR4. Through autophagy activation, exosomes derived from miR-181-5p-modified ADSCs prevented liver fibrosis (34). However, the impacts of miR124-3p on ADSCs has not yet been reported. So, we chose to study miR-124-3p in the current research experiments. In different tissues and cells, miR-124-3p was pervasive, and could modulate number of pathophysiologic processes via targeting mRNAs $(35,36)$. Besides, miR-124-3p was discovered to be upregulated in MSCs mice (37). Hence, miR-124-3p combined with the injection of ADSCs could strengthen ADSCs transplantation by increasing the LPP 


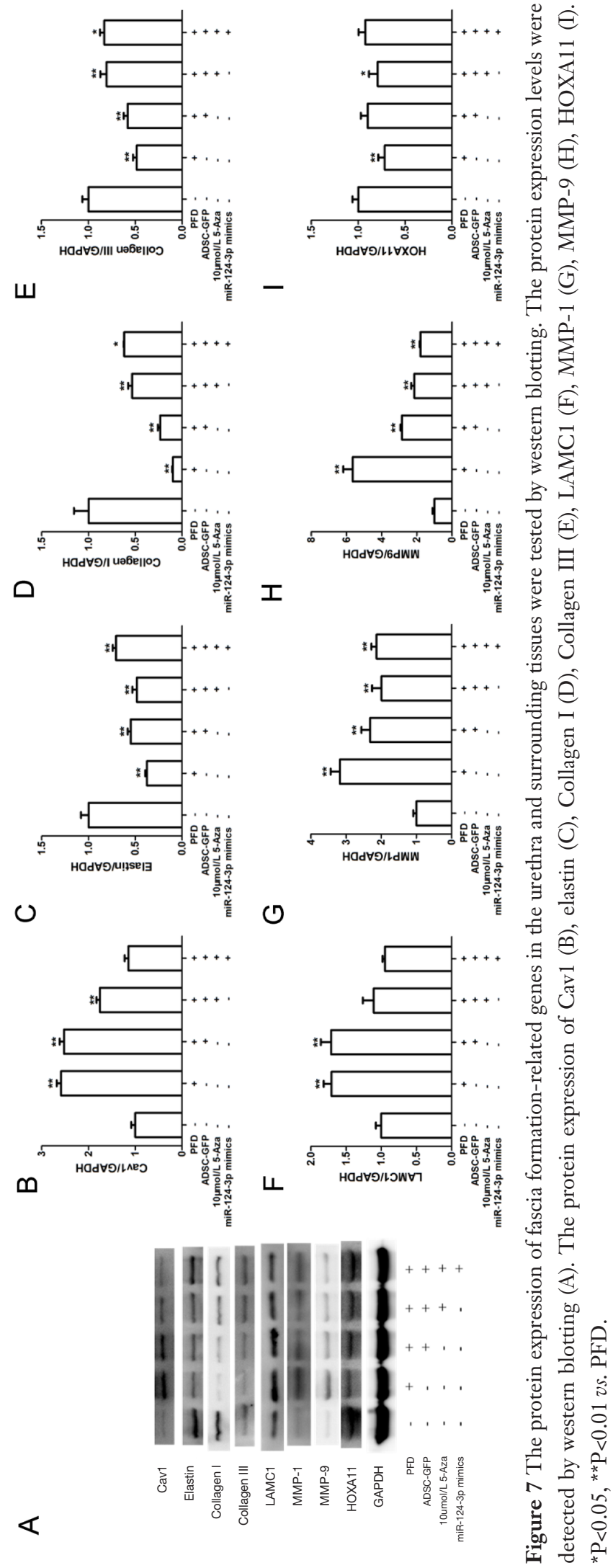

of PFD rats. As a result, miR-124-3p combined with ADSC injection could further repair tissue structures, and tighten the muscular connective tissues and fascia. All the abovementioned functions were affirmed by this experiment.

In most cell types, caveolins, the main component of caveolae, are necessary for small invagination of the plasma membrane (38). Caveolae serve as a signal platform for recruiting a large number of signal molecules, including mitogen-activated protein kinase (MAPK), insulin receptor, and epidermal growth factor receptor (39). As one of the caveolin genes, Cav1 is obviously expressed in adipocytes (40), endothelial cells (41), and smooth muscle cells (40). The genes Cav1 and Cav2 form homo-oligomers or hybrid oligomers and act as scaffold proteins, which bind to signal molecules through their caveolin scaffold domains, thus activating or suppressing signal pathways (42). Through an MAPK pathway-dependent mechanism, Cav1 is crucial in the differentiation of human ADSCs into hepatocyte-like cells (43). However, the deeper mechanisms about the impacts of Cav1 in ADSCs has not yet been elucidated. It has been shown that Cav1 might be a key factor in muscle regeneration (44). By targeting Cav1, miR3074-3p functioned as a positive regulator of myogenic differentiation (23). These previous reports demonstrated that Cav1 may play different roles in myoblasts or ADSCs; meanwhile, Cav1 may associate with some miRNAs. In our paper, we concluded that miR-124-3p facilitated ADSCs via targeting Cav1. Future investigations into the precise mechanisms underlying Cav1-dependent regulation in ADSCs may offer valuable insights into the development of therapeutics for PFD.

Myogenic differentiation of adipose stem cells is tightly modulated by myogenic regulatory factors (MRFs), such as $\mathrm{MyoD}, \mathrm{MyHC}$, and others. In the embryo, and as a fundamental regulator of skeletal muscle lineage determination, MyoD is up-regulated in activated MSCs as an early response to muscle injury $(45,46)$. Sung et al. (47) indicated that MyoD was overexpressed in equine ADSCs, which boosted the potential for myogenic differentiation. During differentiation, the mRNA and protein levels of MyoD1 was reduced by overexpression of miR-483 (48). So far, 8 myosin heavy chain (MyHC) isoforms have been found in mammalian skeletal muscle, but only 4 isoforms are expressed in adult skeletal muscle, namely $\mathrm{MyHC}-$ 1, MyHC-IIa, MyHC-IIb, and MyHC-II (49). The MyHC gene has different expression patterns in different species and different skeletal muscles (50). However, the influence of MyHC in ADSCs has not yet been reported. 
Page 14 of 17
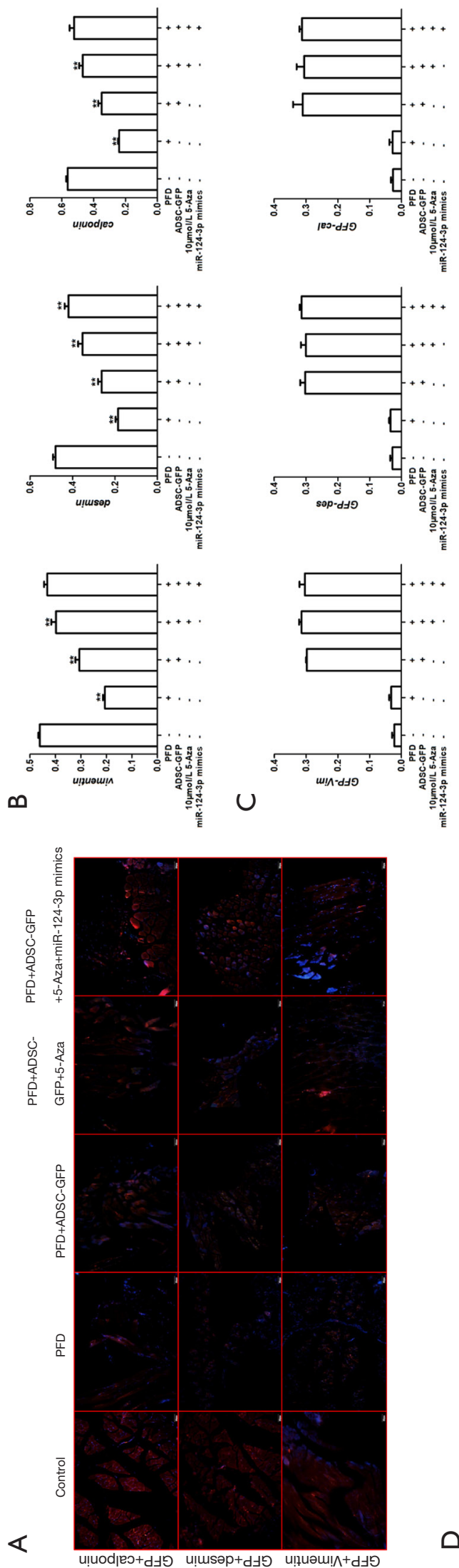

Chen et al. The influences of miR-124-3p/Cav1 on PFD

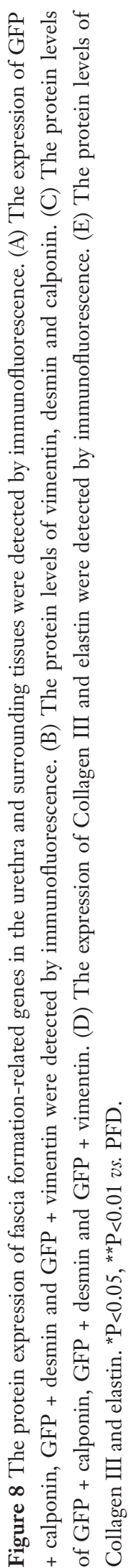


Furthermore, the results of immunofluorescence analysis manifested that the number of $\mathrm{MyHC}$-positive myotubes was decreased by miR-483 (48). In line with the above studies, we discovered that miR-124-3p directly targeted $\mathrm{MyoD}$ and $\mathrm{MyHC}$ to affect its expression on ADSCs.

In addition to MRFs, several other factors are differentially expressed during myogenic differentiation. These factors, such as elastin, Collagen I, Collagen III, HOXA11, LAMC1, MMP1, and MMP9, might emerge as key players in muscle myogenesis by participating in the processes of ADSCs $(51,52)$. Extracellular matrix (ECM) proteins (mainly containing collagen and elastin) have the function of maintaining skin integrity and elasticity, which has become the focus of research for treatment with ADSCs (51). Along with the result of elastin, we found the protein levels of collagen I and III gradually declined in ADSCs. We tested the increases of LAMC1, MMP-1, MMP-9, and HOXA11 mRNA and protein levels. It has been hinted that MMP1 displays collagenolytic activity, MMP-9 degrades elastin, and HOXA11 mainly repressed the expression of MMP1 and MMP-9 $(53,54)$. Consistent with the above research, we discovered that these factors might play vital roles in the development of ADSCs.

In this article, we discovered that miR-124-3p regulated Cav1 expression in ADSCs, thereby enhancing myogenesis and influencing PFD. Taken together, our outcomes suggested that miR-1214-3p may be regarded as a positive regulator of ADSCs through the regulation of Cav1 expression, and may have the potential to accelerate PFD. However, there were some limitations in our paper. This is just a preliminary experiment in the role of miR-124-3p and Cav1 on myogenic differentiation of ADSCs during PFD, and further rescue experimental studies are needed to confirm our findings.

\section{Acknowledgments}

Funding: This research is supported by the following funds: National Natural Science Foundation of China (81971850, LL), Municipal Science and Technology Bureau of Wenzhou (Y20190017, ML), Municipal Science and Technology Bureau of Wenzhou (Y20180148, LL), Municipal Science and Technology Bureau of Wenzhou (Y2020085, ML).

\section{Footnote}

Reporting Checklist: The authors have completed the ARRIVE reporting checklist. Available at http://dx.doi.org/10.21037/ atm-20-8212

Data Sharing Statement: Available at http://dx.doi. org/10.21037/atm-20-8212

Conflicts of Interest: All authors have completed the ICMJE uniform disclosure form (available at http://dx.doi. org/10.21037/atm-20-8212). The authors have no conflicts of interest to declare.

Etbical Statement: The authors are accountable for all aspects of the work in ensuring that questions related to the accuracy or integrity of any part of the work are appropriately investigated and resolved. The experimental protocol of our study was performed in accordance with the Guide for the Care and Use of Laboratory Animals and approved by the First Affiliated Hospital of Wenzhou Medical University.

Open Access Statement: This is an Open Access article distributed in accordance with the Creative Commons Attribution-NonCommercial-NoDerivs 4.0 International License (CC BY-NC-ND 4.0), which permits the noncommercial replication and distribution of the article with the strict proviso that no changes or edits are made and the original work is properly cited (including links to both the formal publication through the relevant DOI and the license). See: https://creativecommons.org/licenses/by-nc-nd/4.0/.

\section{References}

1. Jundt K, Peschers U, Kentenich $\mathrm{H}$. The investigation and treatment of female pelvic floor dysfunction. Dtsch Arztebl Int 2015;112:564-74.

2. Easley DC, Abramowitch SD, Moalli PA. Female pelvic floor biomechanics: bridging the gap. Curr Opin Urol 2017;27:262-7.

3. Wu J, Li J, Chen WK, et al. MicroRNA-214 Affects Fibroblast Differentiation of Adipose-Derived Mesenchymal Stem Cells by Targeting Mitofusin-2 during Pelvic Floor Dysfunction in SD Rats with Birth Trauma. Cell Physiol Biochem 2017;42:1870-87.

4. Hainsworth AJ, Solanki D, Schizas AM, et al. Total pelvic floor ultrasound for pelvic floor defaecatory dysfunction: a pictorial review. Br J Radiol 2015;88:20150494.

5. Faubion SS, Shuster LT, Bharucha AE. Recognition and management of nonrelaxing pelvic floor dysfunction. Mayo Clin Proc 2012;87:187-93. 
6. Fitz FF, Resende AP, Stüpp L, et al. Biofeedback for the treatment of female pelvic floor muscle dysfunction: a systematic review and meta-analysis. Int Urogynecol J 2012;23:1495-516.

7. Wallace SL, Miller LD, Mishra K. Pelvic floor physical therapy in the treatment of pelvic floor dysfunction in women. Curr Opin Obstet Gynecol 2019;31:485-93.

8. Louis-Charles K, Biggie K, Wolfinbarger A, et al. Pelvic Floor Dysfunction in the Female Athlete. Curr Sports Med Rep 2019;18:49-52.

9. Jin M, Wu Y, Wang J, et al. MicroRNA-29 facilitates transplantation of bone marrow-derived mesenchymal stem cells to alleviate pelvic floor dysfunction by repressing elastin. Stem Cell Res Ther 2016;7:167.

10. Alvarez J, Cvach K, Dwyer P. Complications in pelvic floor surgery. Minerva Ginecol 2013;65:53-67.

11. Maher CF, Baessler KK, Barber MD, et al. Surgical management of pelvic organ prolapse. Climacteric 2019;22:229-35.

12. Drost AC, Weng S, Feil G, et al. In vitro myogenic differentiation of human bone marrow-derived mesenchymal stem cells as a potential treatment for urethral sphincter muscle repair. Ann N Y Acad Sci 2009;1176:135-43.

13. Yang B, Li M, Lei H, et al. Low Intensity Pulsed Ultrasound Influences the Myogenic Differentiation of Muscle Satellite Cells in a Stress Urinary Incontinence Rat Model. Urology 2019;123:297.e1-8.

14. Jin M, Chen Y, Zhou Y, et al. Transplantation of bone marrow-derived mesenchymal stem cells expressing elastin alleviates pelvic floor dysfunction. Stem Cell Res Ther 2016;7:51.

15. Tobita M, Tajima S, Mizuno H. Adipose tissue-derived mesenchymal stem cells and platelet-rich plasma: stem cell transplantation methods that enhance stemness. Stem Cell Res Ther 2015;6:215.

16. Arnhold S, Wenisch S. Adipose tissue derived mesenchymal stem cells for musculoskeletal repair in veterinary medicine. Am J Stem Cells 2015;4:1-12.

17. Wang L, Xu L, Xu M, et al. Obesity-Associated MiR-342$3 p$ Promotes Adipogenesis of Mesenchymal Stem Cells by Suppressing CtBP2 and Releasing C/EBP $\alpha$ from CtBP2 Binding. Cell Physiol Biochem 2015;35:2285-98.

18. Kriegsmann M, Randau TM, Gravius S, et al. Expression of miR-146a, miR-155, and miR-223 in formalin-fixed paraffin-embedded synovial tissues of patients with rheumatoid arthritis and osteoarthritis. Virchows Arch 2016;469:93-100.
19. Zhou X, Zhang L, Zheng B, et al. MicroRNA-761 is upregulated in hepatocellular carcinoma and regulates tumorigenesis by targeting Mitofusin-2. Cancer Sci 2016;107:424-32.

20. Singh RP, Massachi I, Manickavel S, et al. The role of miRNA in inflammation and autoimmunity. Autoimmun Rev 2013;12:1160-5.

21. Mens MMJ, Ghanbari M. Cell Cycle Regulation of Stem Cells by MicroRNAs. Stem Cell Rev Rep 2018;14:309-22.

22. Wang J, Liu S, Li J, et al. Roles for miRNAs in osteogenic differentiation of bone marrow mesenchymal stem cells. Stem Cell Res Ther 2019;10:197.

23. Lee B, Shin YJ, Lee SM, et al. miR-3074-3p promotes myoblast differentiation by targeting Cav1. BMB Rep 2020;53:278-83.

24. McCarthy JJ. The MyomiR network in skeletal muscle plasticity. Exerc Sport Sci Rev 2011;39:150-4.

25. Nagle AS, Barker MA, Kleeman SD, et al. Passive biomechanical properties of human cadaveric levator ani muscle at low strains. J Biomech 2014;47:583-6.

26. Li Q, Wang J, Liu H, et al. Tissue-engineered mesh for pelvic floor reconstruction fabricated from silk fibroin scaffold with adipose-derived mesenchymal stem cells. Cell Tissue Res 2013;354:471-80.

27. An Y, Zhao J, Nie F, et al. Exosomes from AdiposeDerived Stem Cells (ADSCs) Overexpressing miR-21 Promote Vascularization of Endothelial Cells. Sci Rep 2019;9:12861.

28. Yu L, Qu H, Yu Y, et al. LncRNA-PCAT1 targeting miR-145-5p promotes TLR4-associated osteogenic differentiation of adipose-derived stem cells. J Cell Mol Med 2018;22:6134-47.

29. Chen DC, Chen LY, Ling QD, et al. Purification of human adipose-derived stem cells from fat tissues using PLGA/silk screen hybrid membranes. Biomaterials 2014;35:4278-87.

30. Wallner C, Huber J, Drysch M, et al. Activin Receptor 2 Antagonization Impairs Adipogenic and Enhances Osteogenic Differentiation in Mouse Adipose-Derived Stem Cells and Mouse Bone Marrow-Derived Stem Cells In Vitro and In Vivo. Stem Cells Dev 2019;28:384-97.

31. Kim SO, Na HS, Kwon D, et al. Bone-marrow-derived mesenchymal stem cell transplantation enhances closing pressure and leak point pressure in a female urinary incontinence rat model. Urol Int 2011;86:110-6.

32. Ryu CM, Yu HY, Lee HY, et al. Longitudinal intravital imaging of transplanted mesenchymal stem cells elucidates their functional integration and therapeutic potency in an 
animal model of interstitial cystitis/bladder pain syndrome. Theranostics 2018;8:5610-24.

33. Guo PY, Wu LF, Xiao ZY, et al. Knockdown of MiR-1405 promotes osteogenesis of adipose-derived mesenchymal stem cells by targeting TLR4 and BMP2 and promoting fracture healing in the atrophic nonunion rat model. Eur Rev Med Pharmacol Sci 2019;23:2112-24.

34. Qu Y, Zhang Q, Cai X, et al. Exosomes derived from miR181-5p-modified adipose-derived mesenchymal stem cells prevent liver fibrosis via autophagy activation. J Cell Mol Med 2017;21:2491-502.

35. Fu W, Wu X, Yang Z, et al. The effect of miR-124-3p on cell proliferation and apoptosis in bladder cancer by targeting EDNRB. Arch Med Sci 2019;15:1154-62.

36. Cai Y, Wang W, Guo H, et al. miR-9-5p, miR-124-3p, and $\mathrm{miR}-132-3 \mathrm{p}$ regulate BCL2L11 in tuberous sclerosis complex angiomyolipoma. Lab Invest 2018;98:856-70.

37. Lu Y, Zhang T, Shan S, et al. MiR-124 regulates transforming growth factor- $\beta 1$ induced differentiation of lung resident mesenchymal stem cells to myofibroblast by repressing Wnt/ $\beta$-catenin signaling. Dev Biol 2019;449:115-21.

38. Lee H, Carnino JM, Jin Y. Caveolin-1 regulates extracellular vesicle-miRNA packaging. Aging 2019;11:8733-5.

39. Parton RG, Tillu VA, Collins BM. Caveolae. Curr Biol 2018;28:R402-5.

40. Fedorenko OA, Pulbutr P, Banke E, et al. CaV1.2 and $\mathrm{CaV} 1.3$ voltage-gated L-type $\mathrm{Ca} 2$ + channels in rat white fat adipocytes. J Endocrinol2020;244:369-81.

41. Kuo A, Lee MY, Yang K, et al. Caveolin-1 regulates lipid droplet metabolism in endothelial cells via autocrine prostacyclin-stimulated, cAMP-mediated lipolysis. J Biol Chem 2018;293:973-83.

42. Lu SY, Rong SS, Wu Z, et al. Association of the CAV1CAV2 locus with normal-tension glaucoma in Chinese and Japanese. Clin Exp Ophthalmol 2020;48:658-65.

43. Guan X, Wang N, Cui F, et al. Caveolin-1 is essential in the differentiation of human adipose-derived stem cells into hepatocyte-like cells via an MAPK pathwaydependent mechanism. Mol Med Rep 2016;13:1487-94.

44. Botzenhart UU, Vaal V, Rentzsch I, et al. Changes in caveolin-1, caveolin-3 and vascular endothelial growth factor expression and protein content after botulinum toxin A injection in the right masseter muscle of dystrophin deficient (mdx-) mice. J Physiol Pharmacol 2017;68:181-9.

45. Yin H, Price F, Rudnicki MA. Satellite cells and the muscle stem cell niche. Physiol Rev 2013;93:23-67.

46. Yamamoto M, Legendre NP, Biswas AA, et al. Loss of MyoD and Myf5 in Skeletal Muscle Stem Cells Results in Altered Myogenic Programming and Failed Regeneration. Stem Cell Reports 2018;10:956-69.

47. Sung SE, Hwang M, Kim AY, et al. MyoD Overexpressed Equine Adipose-Derived Stem Cells Enhanced Myogenic Differentiation Potential. Cell Transplant 2016;25:2017-26.

48. Song C, Yang Z, Dong D, et al. miR-483 inhibits bovine myoblast cell proliferation and differentiation via IGF1/PI3K/AKT signal pathway. J Cell Physiol 2019;234:9839-48.

49. Vrecl M, Cotman M, Uršič M, et al. Age-Dependent Expression of MyHC Isoforms and Lipid MetabolismRelated Genes in the Longissimus Dorsi Muscle of Wild and Domestic Pigs. Animals (Basel) 2018;9:10.

50. Zhang M, Liu YL, Fu CY, et al. Expression of MyHC genes, composition of muscle fiber type and their association with intramuscular fat, tenderness in skeletal muscle of Simmental hybrids. Mol Biol Rep 2014;41:833-40.

51. Guo S, Wang T, Zhang S, et al. Adipose-derived stem cell-conditioned medium protects fibroblasts at different senescent degrees from UVB irradiation damages. Mol Cell Biochem 2020;463:67-78.

52. Shen T, Shen J, Zheng QQ, et al. Cell viability and extracellular matrix synthesis in a co-culture system of corneal stromal cells and adipose-derived mesenchymal stem cells. Int J Ophthalmol 2017;10:670-8.

53. Van Doren SR. Matrix metalloproteinase interactions with collagen and elastin. Matrix Biol 2015;44-46:224-31.

54. Sakowicz A, Lisowska M, Biesiada L, et al. Association of Maternal and Fetal Single-Nucleotide Polymorphisms in Metalloproteinase (MMP1, MMP2, MMP3, and MMP9) Genes with Preeclampsia. Dis Markers 2018;2018:1371425.

(English Language Editor: J. Jones)

Cite this article as: Chen $\mathrm{H}, \mathrm{Li} Z$, Lin $\mathrm{M}, \mathrm{Lv} \mathrm{X}$, Wang J, Wei Q, Zhang Z, Li L. MicroRNA-124-3p affects myogenic differentiation of adipose-derived stem cells by targeting Caveolin-1 during pelvic floor dysfunction in Sprague Dawley rats. Ann Transl Med 2021;9(2):161. doi: 10.21037/atm-208212 